Taiwan, and to provide recommendations for improving the surveillance system.

Methods The reporting system of occupational diseases initiated by Department of Labours was used to retrieve the cases of occupational lung cancer from 2008 to 2014. Descriptive analysis was conducted including identification of exposure to asbestos. We further compared the data with Taiwan Cancer Registry. A review for international comparison of mesothelioma surveillance system was performed.

Results 73 cases of occupational lung cancer were reported, and 42 were suspected to be asbestos-related. 31 cases were confirmed as malignant mesothelioma. Only one of the 42 asbestos-related lung cancer cases was female. Their occupations and industries included construction (36\%), work concerning installation and repair of boilers (24\%), and shipyard and ship breaking (19\%). The year of age at the time of diagnosis is 60.5 , while the induction time was 35.2 years. In the same period, 349 mesothelioma cases were identified in the Taiwan Cancer Registry.

Discussion This study showed that very few mesothelioma patients seek compensation in Taiwan. Further review showed that mesothelioma surveillance system was established in many countries to provide information of mesothelioma epidemic and investigate in asbestos exposure. Some have a specific registry and rely on medical doctor, particularly pathologists, to report. Some directly link the data from the pre-existing cancer registry. In Taiwan, all hospitals were mandated to submit cancer data to the central cancer registry. Improving linkage between mesothelioma surveillance and cancer registry should be considered.

\section{ACCELERATED PNEUMOCONIOSIS BY ALGINATES IN A WORKER IN THE CHEMICAL-PHARMACEUTICAL INDUSTRY. CASE REPORT}

E Frias*, M Valdes. UIDAC, Research Unit, Teaching and Clinical Support in Occupational Health, IMSS, Guadalajara, Mexico

\subsection{6/oemed-2018-ICOHabstracts. 1289}

Introduction Pneumoconiosis is a respiratory disease produced by the inhalation of silicon dioxide, in its crystalline and cryptocrystalline forms, it is common to accompany other powders in the working environment. The accelerated form develops within the first ten years of the beginning of exposure to high silica level, presenting symptoms consisting of chronic cough and dyspnea of exertion accompanied by worsening of radiological images; with a national rate in Mexico of $0.48-$ 2.39 cases per 10000 workers.

Methods 27-year-old female worker, production manager and responsible for the area of alginates in a manufacturing company for the dental industry for 6 years, with inhalation exposure to inorganic powders and alginate vapours whose composition is calcined diatomaceous flow. Begins current disease after entering the alginate area, presenting dry cough, burning sensation in the nose and epistaxis, without going to medical attention. 5 years later she presented dyspnea of great efforts and non-productive cough, evolving at 5 months to dyspnea of moderate efforts and tachycardia; with spirometry that concluded severe restriction. Pneumoconiosis was diagnosed 10 months later by Pneumology, presenting severe dyspnea, tachycardia and nail cyanosis at moderate efforts, requiring the use of home and ambulatory oxygen and treatment with bronchodilators.

Results Symmetrical thorax, decreased respiratory movements, fine rales in the right hemithorax and upper lobe of the left hemithorax, Plethysmography FVC 47\%, TLC 64.4\%, sixminute walk test suspended at 2 min by $\mathrm{SO} 2<85 \%$, oscillometry: increased resistance in distal airway, bronchoscopy with cytopathologic: negative to malignancy, BAAR (-).

Discussion High exposure and no personal protective equipment suitable for alginates within industrial processes can lead to the accelerated development of pneumoconiosis; in the company visit, the inhalation exposure to composite powders with calcined diatomaceous flow was corroborated, which together with the paraclinical studies and the symptomatology presented demonstrated the cause-effect relationship, workinjury.

\section{EARLY DETECTION OF ASBESTOS-RELATED LUNG CANCER BY LOW-DOSE MULTISLICE-CT (LOW-DOSE MSCT)}

V Harth*. Institute for Occupational Medicine and Maritime Medicine (ZAM), University Medical Centre Hamburg-Eppendorf, Hamburg, Germany

\subsection{6/oemed-2018-ICOHabstracts. 1290}

Introduction Lung cancer is the most common cause of death from cancer worldwide, estimated to be responsible for nearly one in five (18\%), or 1.38 million, cancer deaths in 2008. Of all risk factors, smoking has been identified as the major risk factor. Other causes of lung cancer include occupational (e.g. asbestos) and environmental exposures (e.g. radon decay products). Despite the reduction or ban of asbestos use in many countries, the global incidence of asbestos-related lung cancer is still increasing. Nevertheless, asbestos is still produced and exported in some countries in the world. The National Lung Screening Trial (NLST) enrolled persons at high risk for lung cancer to undergo annual screenings with either low-dose CT or single-view posteroanterior chest radiography. In the low CT-group, mortality from lung cancer was reduced by $20.0 \%$. Currently, secondary prevention strategies are extensively discussed to reduce mortality from lung cancer.

Methods In Germany, more than $80 \%$ of lung cancers are diagnosed at an advanced disease stage (clinical stages IIIa, IIIb, and IV) where the survival rate is poor. Since lung cancer is only curable at an early stage of the disease, in Germany, formerly asbestos-exposed insured individuals have the statutory right to receive 'follow-up occupational medical examinations' which target the early detection of asbestosrelated diseases. Recently, the German Social Accident Insurance (DGUV) founded a working group to establish an annual low-dose MSCT scanning program.

Results The eligibility criteria for participants are: at least 10 years of exposure to asbestos (starting before 1985) or a recognised case of asbestos-induced occupational disease (No. 4103 BKV), between 55 and 74 years of age and a history of cigarette smoking of at least 30 pack years. The participants are contacted by GVS (a joint organisation involving all German social accident insurance institutions) or the specific statutory accident insurance and examinations are offered which are carried out locally by selected physicians. A quit-smoking counselling is provided, and participants are asked to donate blood for biomarker research. For MSCT scanning, at least 16 
row scanners are mandatory. The evaluation algorithm follows the recommendation of the National Comprehensive Cancer Network (NCCN Guideline) which specifies interventions according to nodule size. Suspicious findings lead to individual assessment by a pulmonologist and could imply CT control after several weeks, PET imaging, or immediate biopsy. Clinical workup and treatment for malignancies follow the respective guidelines.

Conclusion In Germany, formerly asbestos-exposed insured individuals have the statutory right to receive 'follow-up occupational medical examinations' (secondary prevention). Due to the results of the NLST-Study, the DGUV decided to offer an annual low-dose CT to a highly selected population of former asbestos-exposed workers and workers with asbestos-induced recognised occupational disease. Hereby, we present results of this early detection program.

\section{ASBESTOS RELATED OCCUPATIONAL DISEASES - LEGISLATIVE CHANGES AND INCIDENCE DIFFERENCES IN CROATIA}

Ina Kardoš* ${ }^{*}$ Marina Milaković. Croatian Institute for Health Protection and Safety at Work, Zagreb, Croatia

\subsection{6/oemed-2018-ICOHabstracts. 1291}

Introduction The aim of this study was to identify differences in the occurrence of asbestos-related occupational diseases in relation to the changes in Croatian legislation regarding health protection, surveillance and compensation entitlement due to occupational exposure to asbestos. Aforementioned changes occurred in the period between 2007 and 2008 and are considered as a significant step forward in registering asbestosrelated occupational diseases.

Methods Annual epidemiological and demographic data on newly diagnosed patients with asbestos related diseases have been retrieved from Register of Asbestos Related Occupational Diseases of the Croatian Institute for Health Protection and Safety at Work for the period between 2009 and 2016. Annual Croatian Health Service Yearbooks of the Croatian National Institute for Public Health have been used for obtaining data between 2000 and 2008. Descriptive statistical analysis has been performed.

Results Between 2009 and 20161356 asbestos-related occupational diseases have been diagnosed with peak occurrence in 2011, following: 52\% pleural plaques with asbestosis $(n=705)$, $22.27 \%$ pleural plaques $(n=302), 20.13 \%$ pulmonary asbestosis $(n=273), 4.28 \%$ mesothelioma $(n=58)$ and $1.33 \%$ other respiratory malignant neoplasms $(n=18)$. Between 2000 and 2008104 asbestos-related occupational diseases have been reported (nearly 13 times less compared to the post-legislative period).

Discussion Changes in legislation have, undoubtedly, led to significant differences in the occurrence of occupational asbestosrelated diseases. Reasons for such differences might be partially due to workers' inclination to compensation and indemnification after being diagnosed with occupational disease. However, a relatively late onset of a complete ban of asbestos-engaged work in Croatia is also considered to be a major contributing factor for present results. Considering relatively late peak of occurrence in 2011, it is fair to assume that a significant number of asbestos-related diseases are yet to be reported. Hence, a further need for continuous health surveillance of workers exposed to asbestos cannot be accentuated enough.

\section{SILICA EXPOSURE AND WORK-RELATEDNESS EVALUATION FOR OCCUPATIONAL CANCER IN KOREA}

${ }^{1}$ Hyoung-Ryoul Kim*, ${ }^{2}$ Boowook Kim, ${ }^{1}$ Bum Seak Jo, ${ }^{1} \mathrm{Ji}$-Won Lee. ${ }^{1}$ Dept Occup and Environ Med, College of Medicine, the Catholic University of Korea, Seoul, South Korea; ${ }^{2}$ Occupational Lung Disease Institute, Korea Workers' Compensation and Welfare Service, Incheon, Korea

\subsection{6/oemed-2018-ICOHabstracts. 1292}

Introduction Crystalline silica has been classified as a definite carcinogen (Group 1) causing lung cancer by the International Agency for Research on Cancer (IARC). In Korea, crystalline silica has been the most common causal agent for workers to apply to the Korea Workers' Compensation and Welfare Service (KWCWS).

Methods We used KWCWS data to evaluate workers' crystalline silica exposure levels according to their occupations and industries, and reviewed research papers describing the doseresponse relationship between cumulative exposure levels and lung cancer incidence. In addition, we reviewed lung cancer cases accepted by the KWCWS, and suggest new criteria for defining occupational cancer caused by crystalline silica in Korea.

Results A review of 120 cases of occupational lung cancer confirmed through an epidemiological survey of the KWCWS since 2007 revealed that 45 cases (37.5\%) involved miners. Most of the coincidental exposures (72.5\%) involved asbestos, diesel exhaust particles, radon, hexavalent chromium, and so on. From the carcinogenic exposure to the onset of the disease, there was no case of less than 10 years, and the lag time was more than 40 years in $40 \%$ of the cases. Among the patients whose cases were approved, 19.2\% had pneumoconiosis, while many cases were approved without pneumoconiosis. A high level of exposure was found in the construction industry, and significant exposures were also confirmed among miners and foundry workers. Stone quarrying and stone laying were also found to expose workers to high concentrations of crystalline silica.

Discussion Rather than confining to miners, we propose recognising occupational lung cancer whenever workers with pneumoconiosis develop lung cancer, regardless of their industry. Coincidental exposure and lag time should also be considered in evaluations of work-relatedness.

\section{AN ANALYSIS OF LATENT PERIOD AND SURVIVAL FACTORS OF KOREAN PATIENTS WITH MALIGNANT MESOTHELIOMA}

${ }^{1}$ Sung Soo Lee*, ${ }^{1}$ Eun Chul Jang, ${ }^{2}$ Dong \| kim, ${ }^{3}$ Dongmuk Kang, ${ }^{1}$ Yong Jin Lee. ${ }^{1}$ Soonchunhang University, Chunan, Korea; ${ }^{2}$ Seonam University, Seoul, Korea; ${ }^{3}$ Pusan University, Pusan, Korea

\subsection{6/oemed-2018-ICOHabstracts. 1293}

Introduction Malignant mesothelioma is an aggressive tumour occurring in mesothelioma cells of pleura and peritoneum. About $80 \%$ of malignant mesothelioma cases are known to be caused by asbestos. Malignant mesothelioma is known to have a very poor prognosis with an average survival period of about 12 months. The number of patients of malignant 